\title{
EDITORIAL
}

\section{Screening: the need for a balance}

New tests and methods of ophthalmic examination have increased our ability to detect disease at earlier stages, or risk factors for such disease in asymptomatic subjects. This has raised the possibility that earlier detection and treatment might prevent later devastating visual loss. Screening, or the identification of asymptomatic subjects who are at high risk of clinically overt disease, has been advocated for a whole range of ophthalmic conditions including cataract, glaucoma, diabetic retinopathy and amblyopia. Stannard et al ${ }^{1}$ have suggested yet another condition, retinopathy of prematurity (ROP) for active screening, If all the suggested recommendations for screening were taken up, ophthalmic services would be overwhelmed. How can we decide which conditions merit screening, and in what groups screening is warranted?

Some of the confusion over recommendations may lie in failure to distinguish clearly between two separate purposes of screening. The term screening is used loosely in the sense of surveillance, as a research tool to identify prevalence and incidence of conditions or risk factors, and their outcome. As with any research, this does not promise benefits to the subjects involved. Recommendations to screen individuals in clinical practice, however, imply the second context, the promise of earlier detection and treatment of disease, and hence, benefit to the individual screened.

Wilson and Jungner formulated principles of screening over 20 years ago ${ }^{2}$. Briefly, these are that the condition sought should pose an important health problem, the natural history of the disease should be well understood, there should be a recognisable early stage and treatment at this early stage should be of more benefit than treatment started at a later stage. There should be a suitable test, acceptable to the population screened, adequate facilities for the diagnosis and treatment of abnormalities detected, and for diseases of insidious onset, screening should be repeated at intervals determined by the natural history of the disease. The chance of physical or psychological harm to those screened should be less than the chance of benefit and the cost of the screening programme should be balanced against the benefit it provides.

As an example, how well does ROP meet these criteria? A recent randomised trial of cryotherapy for severe stage 3 neovascular ROP demonstrated improved outcome, defined as absence of three anatomic features of abnormality, in eyes which were treated compared with eyes which received no treatment ${ }^{3}$. The absolute benefits of cryotherapy in the 156 eyes treated in this trial were an estimated 33 eyes $(22 \%$ reduction $\times 156)$ prevented from unfavourable anatomic outcome, or a possible 17 eyes prevented from blindness (assuming 50\% probability of blindness in eyes with these abnormal anatomic changes). The adverse effects of cryotherapy included 24 subjects with intraoperative ocular complications, and 21 subjects with systemic complications including bradycardia, asystole and cyanosis. That is, to prevent one possible case of blindness about 9 eyes were treated with cryotherapy with 1-2 of these having intraoperative complications, and 1-2 of these having systemic complications.

Another difficulty is deciding the threshold for treatment in asymptomatic subjects. Extrapolation of benefit from treatment trials with clearly defined severe criteria to treatment at a less severe stage may not be appropriate. Stannard et al., have highlighted the lack of knowledge of the natural history of retinopathy: in their series, they reported that $18 / 28$ babies with stage 1 ROP spontaneously regressed and developed morphologically normal retinal vascularisation and three babies with bilateral stage 2 ROP spontaneously resolved. Even of the seven babies who developed stage 3 disease (neovascular ridge with extra retinal new vessel proliferation) two who did not have cryotherapy both subsequently developed normal retinal vascularisation. Stannard et al., had a lower threshold for cryotherapy than the multicentre trial; the only baby of the seven considered eligible for treatment using trial criteria had the worst outcome.

Screening and treatment programmes should also take into account not only benefits and risks in those eligible for treatment but all those undergoing the screening procedure. In the 
cryotherapy trial, the criteria for screening were subjects weighing less than 1251 grams, surviving to 28 days without fatal anomalies or other eye problems. 3862 infants had to be screened to identify 291 as eligible for cryotherapy. This implies that 3571 infants, or 12 out of every 13 infants screened derived no benefit from the screening programme. Similarly, Stannard et al., using wider criteria, had to screen 169 babies regularly to find 7 babies with stage 3 disease, possibly eligible for cryotherapy, i.e. for every treatment eligible baby detected, 24 babies had to be screened for no benefit. Neither study quantified the incidence of morbidity associated with the screening procedure itself, which was indirect ophthalmoscopy and scleral depression repeated regularly where indicated, but as Stannard et al. describe clearly, this is not negligible. Problems include systemic effects of mydriatics such as tachycardia, vomiting and ileus, and stress of ophthalmoscopy such as stimulation of the oculocardiac reflex causing bradycardia, or cardiorespiratory complications in manoeuvring ventilated babies: these complications may be particularly dangerous in fragile preterm newborn. Where benefit of screening and treatment is based on a probability of detecting and preventing, rather than improving, an actual clinical outcome such as visual loss it is much harder to justify any adverse effects, and particularly important to document and quantify them.

ROP highlights many of the difficulties inherent in any screening programmes. There is tremendous pressure for ophthalmologists to undertake screening for a whole range of conditions, and it would be well to consider how many of these conditions fit the criteria of Wilson and Jungner. While in ophthalmology, most diseases may satisfy the first criterion, that is, visual loss is serious, what is less clear is the evidence that early detection and treatment leads to benefit for the individual, let alone the magnitude of the benefit. Even less well understood are the potential dysbenefits for the normal individuals who may have to undergo the screening test, and the individuals who are misdiagnosed and undergo treatment. Evaluation of screening must take into account the outcome not only in those individuals identified by the screening technique for the treatment, but the outcome in all subjects undergoing screening. When deciding whether to institute a screening programme, one must consider not only the final clinical goal, in this case, reduction of visual loss, but also possible adverse effects of the screening and treatment procedures. These benefits and risks should be stated not just in relative, i.e. percentage terms, but absolute terms, i.e. how many screened, how many treated, how many suffer adverse effects, to prevent one case of visual loss.

In this context, an example from cardiovascular disease may be of interest: randomised controlled trials undoubtedly show a benefit of treatment of high blood pressure of $20 \%-40 \%$ reduction in clinical events. For mild hypertension, i.e. diastolic pressure $95-109 \mathrm{mmHg}$, this $40 \%$ benefit is of the absolute order of 850 subjects treated for one year to prevent one stroke ${ }^{4}$; whether this benefit is sufficiently worthwhile to justify treating all mild hypertensives is still debated. This is analogous to the difficulties over treatment of raised intraocular pressure at various levels to prevent glaucomatous visual loss.

For most conditions we need much more information about the incidence, natural history, sensitivity, specificity and predictive value of any screening test, and effects of treatment, and this information can only be achieved by properly conducted longitudinal studies and trials. Large randomised controlled trials of all possible screening programmes are not always feasible so what do we do? At a minimum, estimates of the absolute magnitude of potential benefit of screening and treatment, dysbenefits and costs should be attempted using existing data before recommending any screening policy. Ultimately, the decision to screen depends on judgements of two issues: the balance of possible costs and benefits for the individual and the optimal use of limited resources. These judgements are not universal. However, making explicit our assumptions might improve our clinical judgements and practice.

Kay-TeE KhaW, University of Cambridge School of Clinical Medicine, Addenbrookes Hospital, Cambridge CB2 2QQ 


\section{References}

${ }^{1}$ Stannard KP, Mushin AS, Gamsu HR. Screening for retinopathy of prematurity in a regional neonatal intensive care unit. Eye 1989, 3: 371-378.

2 Wilson JMG, Jungner G. Principles and practice of screening for disease. World Health Organisation. WHO Public Health Paper, 34, 1968.

${ }^{3}$ Cryotherapy of Retinopathy of Prematurity Cooperative Group. Multicenter trial of cryotherapy for retinopathy of prematurity: preliminary results. Paediatrics 1988,$81 ; 697-706$.

${ }^{4}$ Medical Research Council Working Party. MRC trial of treatment of mild hypertension: principal results. British Medical Journal 1985, 291: 97-104. 